\title{
Inter-Linkage of Transports and its Bridging Mechanism
}

\author{
Katsumi IDA \\ National Institute for Fusion Science, Toki, Gifu 509-5292, Japan \\ (Received 13 November 2007 / Accepted 13 March 2008)
}

\begin{abstract}
The physical mechanisms determining the diffusive and non-diffusive terms of particle, momentum and heat transports are described. The non-diffusive term in particle transport, which causes inward pinch or outward flux, is driven by the temperature gradient and the magnetic field curvature. The non-diffusive term in the momentum transport, which drives spontaneous toroidal rotation, is found to depend on the electric field and ion temperature gradient in the plasma. In heat transport, no clear non-diffusive term is observed. The temperature and temperature gradient dependences of the diffusive terms are discussed.
\end{abstract}

(C) 2008 The Japan Society of Plasma Science and Nuclear Fusion Research

Keywords: transport, Reynolds stress, tokamak, stellarator

DOI: $10.1585 /$ pfr.3.S1003

\section{Introduction}

The transports in a magnetically confined plasma are complicated because of the inter-linkage between particle momentum, and heat radial fluxes. In general, the radial fluxes of particle, momentum, and heat have diffusive terms, in which the radial fluxes are proportional to the gradients of density, momentum, and heat, and non-diffusive terms as offsets of radial fluxes. Since the non-diffusive terms are driven by the gradients of other plasma parameters, in the transport matrix, they are categorized as offdiagonal terms, while the diffusive terms are categorized as diagonal terms. The existence of the non-diffusive term of transport has been recognized in particle transport (and impurity transport) as the Ware pinch by the toroidal electric field [1] or convection by ion temperature $\left(T_{\mathrm{i}}\right)$ gradientdriven turbulence [2]. The peaked profile of the electron density observed in the steady-state with gas puff fueling clearly shows the significant contribution of the nondiffusive inward particle flux, because the particle source is localized near the plasma periphery. The inward pinch observed in a stellarator, where there is no toroidal electric field, suggests that turbulence-driven convection can easily overcome thermodiffusion [3]. The non-diffusive term in the momentum transport was found experimentally [4-6], and it appears as a spontaneous toroidal rotation [7]. The significant contribution of the non-diffusive term to the transport shows a strong inter-linkage of the transport between particle, (poloidal and toroidal) momentum, and (ion and electron) heat transports.

In the plasmas with a transport barrier, where a strong gradient is produced, the inter-linkage of the transports becomes more significant. (A good example is a large spontaneous toroidal rotation at the internal transport barrier $[8,9])$. This is because these non-diffusive terms are correlated to the gradients of other plasma parameters (ion

$\overline{\text { author's e-mail: ida@nifs.ac.jp }}$ temperature gradient for particle transport or potential gradient for momentum transport). Therefore, these nondiffusive terms are considered to be off-diagonal terms of the so-called transport matrix. Turbulence transport theory has explored the physics bridging mechanism for the off-diagonal terms of the transport matrix [10]. Recently, a theoretical model of symmetry breaking of turbulence by radial electric field shear [11] was proposed to explain the strong correlation between non-diffusive momentum flux and ion pressure gradients (and also radial electric field) observed in experiments. In this paper, non-diffusive terms of particle and momentum transports and diffusive terms of heat transport are discussed based on experiments in stellarators and tokamaks.

\section{Diffusive and Non-Diffusive Terms in Transports}

It is well known that the non-diffusive term has a significant contribution in the radial flux of particles and that it causes the inward flux that is necessary to achieve a peaked density profile with a particle source localized near the plasma edge. Due to the role of spontaneous toroidal rotation in magneto-hydro-dynamic (MHD) stability, the non-diffusive term of momentum transport has been highlighted in recent momentum transport studies. On the other hand, the non-diffusive term of heat transport has not been clearly identified, although there were experimental results that suggest the heat pinch. Figure 1 shows the physics mechanism determining the diffusive term and the nondiffusive terms of the radial flux of particle, momentum, and energy transports. The radial profiles of density in the plasma can not be explained by the diffusion process, because the density profiles are peaked at the plasma center, although the particle source is localized near the plasma edge. In order to explain the peaked density profiles, in- 


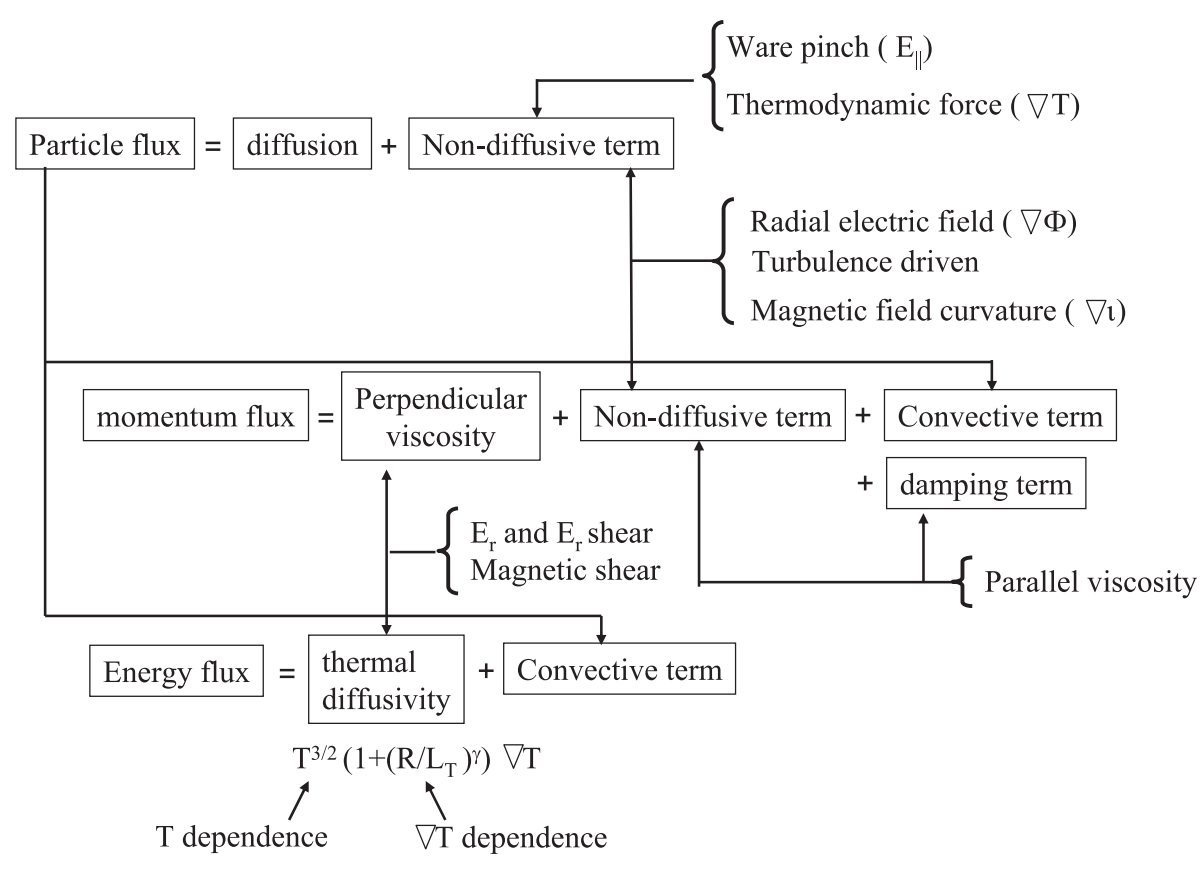

Fig. 1 Physics mechanism determining the diffusive term and the non-diffusive terms of the radial flux of particle, momentum, and energy transports.

ward particle pinch has been introduced. This particle pinch is considered to be a non-diffusive term in particle transport. There are various physics mechanisms contributing to the non-diffusive terms in the particle transport. The inward pinch driven by the toroidal electric field is well known as the Ware pinch in tokamaks. However, the inward pinch observed in steady-state plasmas in stellarators can not be explained by the Ware pinch, because there is no toroidal electric field in these experiments. In stellarator plasmas, the thermodynamic force driven by the electron temperature $\left(T_{\mathrm{e}}\right)$ gradient contributes to significant outward flux. This term has been confirmed experimentally in stellarators.

It is well known that momentum and energy transport have convection terms due to the radial flux of particles. In general, these convective terms are relatively small. In addition to the convective term, the non-diffusive term of momentum transport was found in the 1990's. More recently, the toroidal rotation driven by this non-diffusive term is recognized as a spontaneous toroidal rotation, because a finite toroidal rotation can exist even for the zero momentum radial fluxes (zero momentum source), if the non-diffusive term is balanced with the diffusive term. Although there are significant non-diffusive terms in the particle and momentum transports (identified as inward pinch and spontaneous toroidal rotation), there is no clear evidence of a nondiffusive term in the energy flux of heat transport. It is interesting that the radial electric field and the magnetic field curvature affect both the non-diffusive terms of particle and momentum transports, while the radial electric field and its shear affect both the diffusive terms of momentum and en- ergy transports. Therefore, particle, momentum, and heat transport are coupled to each other through these physics elements.

\section{Particle Transport}

The diffusive term of particle transport has been derived from time-dependent transport analysis of a discharge with gas puff modulation. Figure 2 (a) shows the temperature dependence of the diffusion coefficient evaluated in the Large Helical Device (LHD) [12]. The measured diffusion coefficient is larger than that predicted by neoclassical theory by one order of magnitude. Both in the core and the edge, the diffusion coefficient has an electron temperature dependence of $\alpha \approx 1$, where $D \propto T_{\mathrm{e}}^{\alpha}$, which is weaker than that of the neoclassical prediction ( $\alpha \approx 2.6$ ). Figure 2(b) shows the relationship between the normalized temperature and density gradients experimentally obtained in Wendelstein 7AS. Since both the density and temperature gradients contribute to the outward flux, there should be an inward flux to sustain the steady-state density profile where $\Gamma \approx 0$. The particle flux can be written as $\Gamma=-n_{\mathrm{e}}\left(D_{11} \nabla n_{\mathrm{e}} / n_{\mathrm{e}}+D_{12} \nabla T_{\mathrm{e}} / T_{\mathrm{e}}-u\right)$ where $D_{11}$ and $D_{12}$ are the on- and off-diagonal elements of the transport matrix and the velocity $u$ is a convective contribution. The ratios of these coefficients derived from the slope of the data plotted in Fig. 2 (b) are $D_{12} / D_{11} \approx 1.4$ and $u / D_{11} \approx-12.5 \mathrm{~m}^{-1}$, respectively. The significant contribution of the inward term of $u / D_{11}$ overcomes the outward term of $D_{12} / D_{11}$ of the non-diffusive term as well as the diffusive term and sustains the peaked density profile in 
(a)

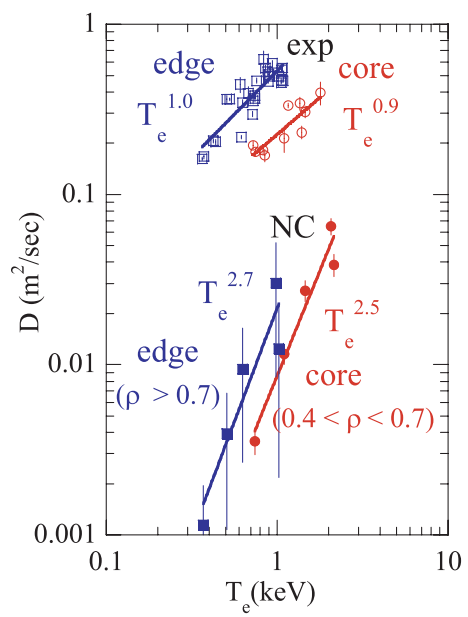

(b)

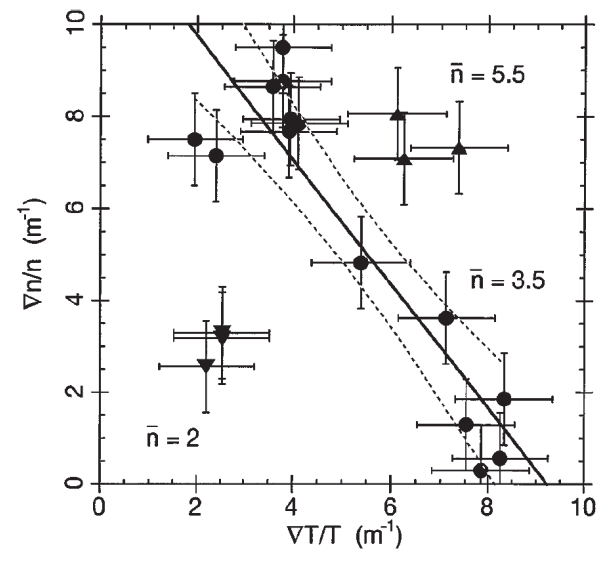

Fig. 2 (a) Temperature dependence of the diffusion coefficient in the core $(0.4<\rho<0.7)$ and edge $(\rho>0.7)$ experimentally evaluated and neoclassically predicted in LHD [12]. The core and edge values are estimated from the average value in the core $(0.4<\rho<0.7)$ and edge $(0.7<\rho<1.0)$ at $R_{\mathrm{ax}}=3.6 \mathrm{~m}, B_{\mathrm{t}}=2.75,2.8 \mathrm{~T}$. (b) The relationship between normalized temperature and density gradient estimated between $r=2$ and $8 \mathrm{~cm}$ for three densities in Wendelstein-7AS [3]. For $\Gamma \approx 0$, it follows that $D_{12} / D_{11} \approx 1.4$ and $u / D_{11} \approx-12.5 \mathrm{~m}^{-1}$.

the steady state. Because various physics mechanisms contribute to the non-diffusive term of particle transport, it is still open to question which physics mechanisms become dominant. A more comprehensive understanding should be sought from the comparison of density profiles in stellarators (typically flat or hollow) with those in tokamaks (typically peaked).

Similar experiments have been performed in steadystate fully noninductive current plasmas in tokamaks to eliminate the contribution of the Ware pinch due to toroidal electric field to drive ohmic current. Figure 3 shows the relationships between $\nabla n_{\mathrm{e}} / n_{\mathrm{e}}, \nabla T_{\mathrm{e}} / T_{\mathrm{e}}$, and $\nabla q / q$ in the Tore Supra tokamak. As seen in Figs. 3(a) and (b), there are two distinguishable regions $(\rho<0.3$ and $0.35<\rho<0.6$ ) that show different slopes [13]. According to the turbulent theory [14,15], turbulent thermodiffusion can generate a pinch velocity, inward or outward, proportional to the inverse of electron gradient length. In general, since the temperature gradient is negative, the thermo-diffusion force produces the outward flux by the collision process in the neoclassical transport. However, when the turbulence transport becomes dominant, the particle fluxes are determined by the phase relationship between the density and potential fluctuations driven by the temperature gradients; the particle flux can be inward for a negative temperature gradient if the turbulence-driven term is dominant. The direction of the thermodiffusion flux is found to change when moving from the outer to the inner plasma. The sign of the thermodiffusion pinch is determined by the slope. This experiment shows that thermo-diffusion can be inward $(\rho<0.3)$ or outward $(0.35<\rho<0.6)$ depending on the plasma radius, where the unstable mode (ITG or ETG) is expected to be different because of the change in the ratio of $\nabla T_{\mathrm{e}} / \nabla T_{\mathrm{i}}$. In the region of $\rho=0.35-0.6$, where the thermo-diffusion flux is outward, a physics mechanism driving the inward pinch is necessary in order to sustain the sharp density gradient in the steady state. The clear relationship between $\nabla n_{\mathrm{e}} / n_{\mathrm{e}}$ and $\nabla q / q$ in Fig. 3(c) strongly suggests that the magnetic field curvature $(\nabla q / q$, where $q$ is a safety factor) drives the inward pinch $[16,17]$. It is interesting that the sign of the magnetic field curvature-driven flux in stellarators is opposite to that in tokamaks, and that the non-diffusive term becomes outward flux in the plasma core. However, the existence of the inward convection is clearly observed experimentally and is necessary to explain the sharp gradient of electron density near the plasma edge. It is still open to question how the physics mechanism affects the inward flux near the plasma edge in sterllarators, where there is no Ware pinch and both the thermo-diffusion flux and the flux driven by the magnetic field curvature are expected to be outward because $\nabla T_{\mathrm{e}} / T_{\mathrm{e}}<0$ and $\nabla q / q<0$.

In neoclassical theory, the impurity density profiles are expected to be more peaked than the bulk ions because the density gradient of impurities should be larger than the density gradient of bulk ions by a factor of ion charge $Z$. This is because both the impurity and bulk ions satisfy the radial force balance and the rotation velocity of impurity, and bulk ions tend to be equal due to the friction between impurity and bulk ions. Because the impurity has a higher ion charge, $Z$, impurity transport is more sensitive to the radial electric field than bulk particle transport. The positive radial electric field in the electron root tends to drive outward flux and suppress the accumulation of impurities and radiation collapse $[18,19]$. It is a crucial issue to achieve poor impurity confinement (impurity exhaust) and good energy confinement, simultaneously. In stellarators, poor 
(a)

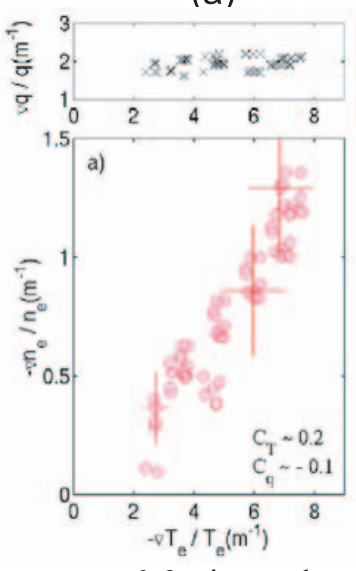

$\rho<0.3$ : inward (b)

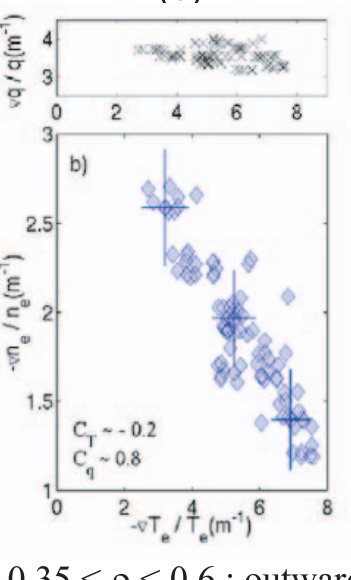

(c)

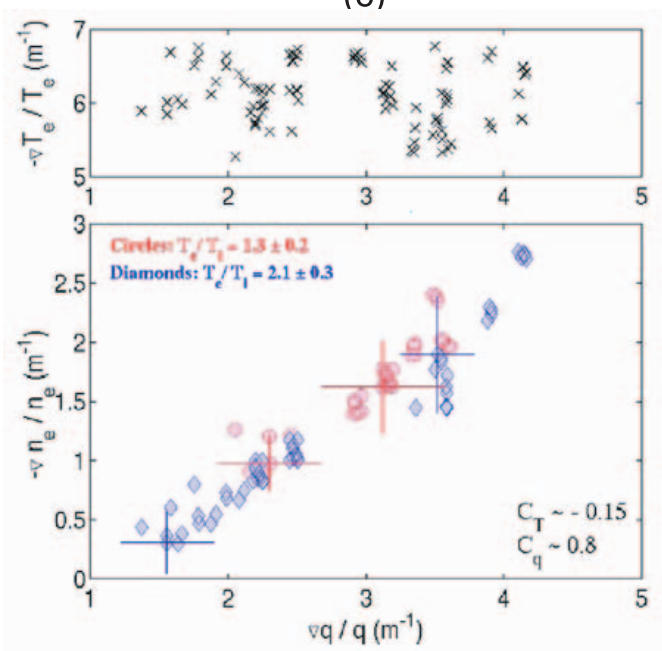

Fig. $3 \nabla n_{\mathrm{e}} / n_{\mathrm{e}}$ versus $\nabla T_{\mathrm{e}} / T_{\mathrm{e}}$ from a set of seven discharges in Tore Supra tokamak: (a) for $r / a \leq 0.3, T_{\mathrm{e}} / T_{\mathrm{i}}=2 \pm 0.4, \nabla T_{\mathrm{e}} / T_{\mathrm{e}}=3.8-4.8$; (b) for $0.35 \leq r / a \leq 0.6, T_{\mathrm{e}} / T_{\mathrm{i}}=1.2 \pm 0.4, \nabla T_{\mathrm{e}} / T_{\mathrm{e}}=0.7-3.5$. Corresponding variation of $\nabla q / q$ is displayed at the top, where $q$ is a safety factor. (c) $\nabla n_{\mathrm{e}} / n_{\mathrm{e}}$ versus $\nabla q / q$ within $0.3 \leq r / a \leq 0.6$ from a set of seven discharges, keeping $\left|\nabla T_{\mathrm{e}} / T_{\mathrm{e}}\right|$ at the values of $6 \mathrm{~m}^{-1} \pm 10 \%$ and $\nabla T_{\mathrm{e}} / T_{\mathrm{e}}=0.6 \pm 0.3[13]$.

(a)

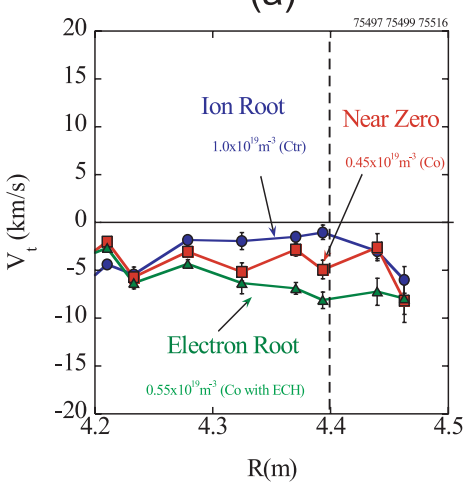

(b)

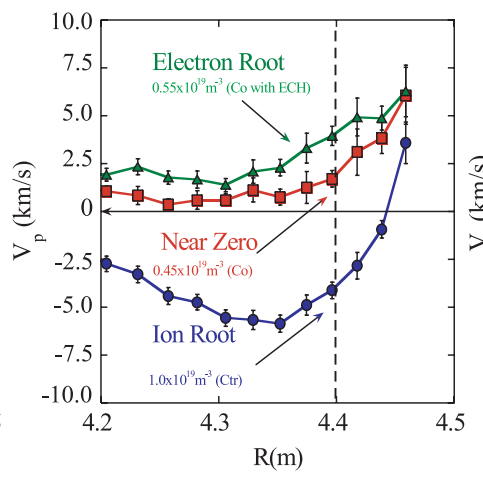

(c)

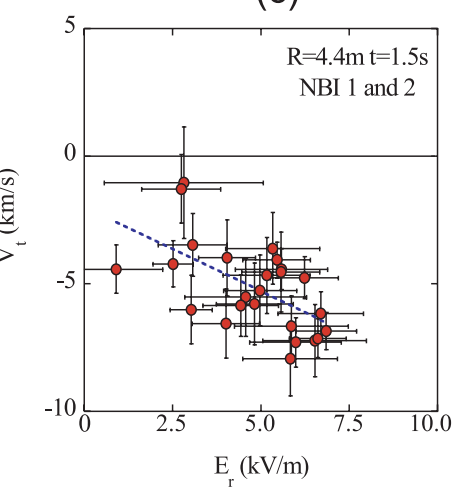

Fig. 4 Radial profiles of (a) toroidal and (b) poloidal rotation velocities, and (c) relationship between toroidal and poloidal rotation velocities in LHD [23].

impurity confinement is observed in plasmas with good energy transport; for example, the HDH mode in Wendelstein 7-AS [20] and the electron ITB and high-ion-temperature mode in LHD.

\section{Momentum Transport}

Since the damping of the plasma toroidal velocity owing to parallel viscosity is significant near the plasma periphery in stellarators [21], toroidal rotation near the plasma edge is determined by the radial electric field, while the core toroidal rotation is determined by the momentum from the tangential NBI. The momentum flux including the non-diffusive term due to the radial electric field can be expressed as $P_{\phi}=-m n\left(\mu_{\perp} \nabla V_{\phi}-\xi E_{r} / B_{\theta}\right)$ [22]. In order to investigate the effect of the radial electric field on the toroidal rotation, a density scan is performed near the threshold of the transition from the ion root (negative $E_{r}$ ) to electron root (positive $E_{r}$ ). In LHD, the transition from the ion root to the electron root takes place at low density, where the plasma is well into the collisionless regime $\mu_{\mathrm{e}}^{*}<0.1$, because of the non-ambipolar particle flux, as predicted by neoclassical theory. As seen in Fig. 4, the plasma rotates more in the "counter" direction (anti-parallel to equivalent plasma current) for positive $E_{r}$, while it rotates more in the "co" direction for negative $E_{r}$ [23]. The relationship between the toroidal rotation and the radial electric field is plotted in Fig. 4(c). This result shows that the positive radial electric field drives the toroidal rotation in the counter direction, where the toroidal rotation contributes to the negative radial electric field. It should be emphasized that the non-diffusive term is comparable or even larger than the diffusive term in the momentum transport, and that the direction of the toroidal flow due to the non-diffusive 
(a)

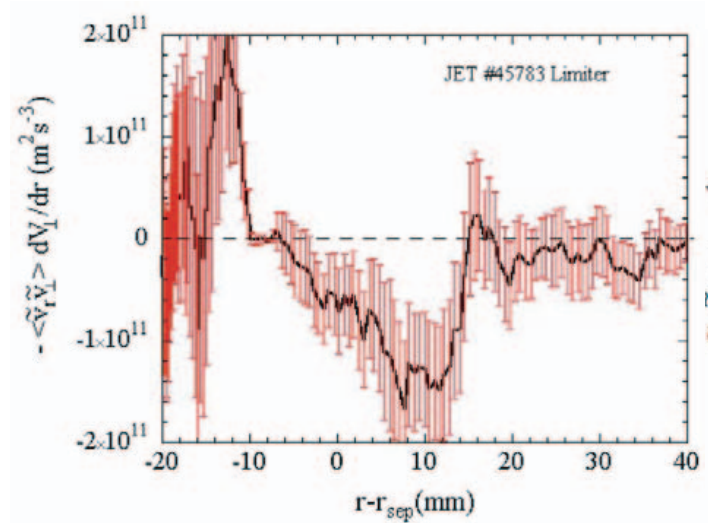

(b)

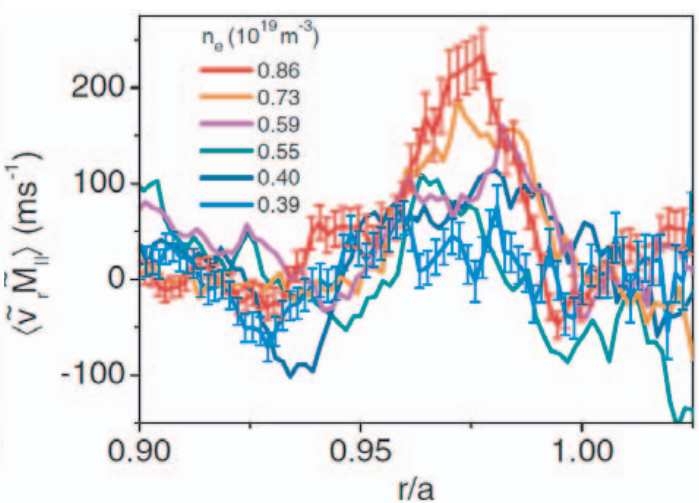

Fig. 5 Radial profile of the (a) radial-perpendicular Reynolds stress component in the plasma boundary region in the JET tokamak and the (b) radial-parallel Reynolds stress component in the TJ-II plasma boundary region at different plasma densities. Parallel velocity is quantified by the parallel Mach number [24,25].

term is anti-parallel to the direction of the $\left\langle E_{r} \times B_{\theta}>\right.$ drift. This is in contrast to the spontaneous flow in tokamaks, where the direction of the toroidal flow is parallel to the direction of the $<E_{r} \times B_{\theta}>$ drift, because the toroidal viscosity is nearly zero due to the toroidal symmetry.

The spontaneous toroidal flow becomes more significant in plasmas with an internal transport barrier, where large electric fields are observed. In CHS, a spontaneous toroidal flow overcomes the toroidal flow driven by co$\mathrm{NBI}$, and the plasma rotates in the counter direction associated with the formation of a large positive $E_{r}$. In contrast, the plasma rotates in the counter direction at the ITB region where a strong negative radial electric field is produced in JT-60U. The significant difference between tokamak and heliotron devices is in the relationship between the poloidal field direction and the direction of the dominant symmetry. The pitch angle of the dominant symmetry is even larger than that of the averaged poloidal field in a heliotron device, while it is zero due to the toroidal symmetry in tokamaks.

In a stellarator, turbulence in the plasma as well as non-ambipolar flux can drive flows perpendicular $(E \times B)$ and parallel (spontaneous toroidal flow) to the magnetic field through Reynolds stress. Experiments to investigate the turbulence-driven $E \times B$ flow and the spontaneous toroidal flow have been performed in the vicinity of the last closed flux surface (LCFS) in JET and in TJ-II. The radialperpendicular component of the production term has been investigated in the vicinity of LCFS in the JET tokamak, as seen in Fig. 5(a) [24]. The Reynolds stress perpendicular to the magnetic field is evaluated from the coupling of fluctuations of radial velocity and $E \times B$ velocity measured with probes in JET. As seen in Fig. 5(a), the significant Reynolds stress is observed near the separatrix of the plasma $\left(\left|r-r_{\text {sep }}\right|<1 \mathrm{~cm}\right)$, which would be clear experimental evidence of turbulence-driven poloidal rotation in tokamaks. Figure 5(b) shows the radial structure of the cross- correlation between parallel and radial fluctuating velocities in the proximity of the LCFS in the TJ-II stellarator [25]. The level of cross-correlation $\left\langle v M_{\|}\right\rangle$increases for plasma densities above the threshold value to generate $E \times B$ sheared flows. The appearance of gradients on $<v M_{\|}>$is due to both radial variations in the level of fluctuations and in the cross-phase correlation between fluctuating radial and parallel velocities. It has been found that the energy transferred from dc flows to turbulence, directly related to the momentum flux and the radial gradient in the perpendicular flow, can be both positive and negative in the proximity of sheared flows. Furthermore, the energy transfer rate is comparable with the mean flow of kinetic energy normalized to the correlation time of the turbulence, implying that this energy transfer is significant. These results show that turbulence can act as an energy sink or energy source for the mean flow near the shear layer.

\section{Energy Transport}

There is no clear evidence of a non-diffusive term of the energy transport except for a few experiments in plasmas with electron cyclotron heating (ECH) [26]. In most cases, the diffusive term only can explain the energy transport observed in experiments. The diffusive term of energy transport is evaluated as thermal diffusivity (ratio between heat flux normalized by density and the temperature gradient). In general, the heat flux depends on the temperature and the temperature gradient, and it is non-linear. Therefore the thermal diffusivity has a temperature dependence and also a temperature gradient dependence in the non-linear regime. Figure 6 shows the $\nabla T_{\mathrm{e}}$ dependence of $\chi_{\mathrm{e}}$ (obtained from the static analysis, i.e., the power balance analysis) in JT-60U, Wendelstein 7-AS, and LHD. In Fig. 6, the thermal diffusivity is normalized by $T_{\mathrm{e}}^{3 / 2}$, which is predicted by Gyro-Bohm transport, in order to eliminate the $T_{\mathrm{e}}$ dependence of $\chi_{\mathrm{e}}$ and make the $\nabla T_{\mathrm{e}}$ dependence 
(a)

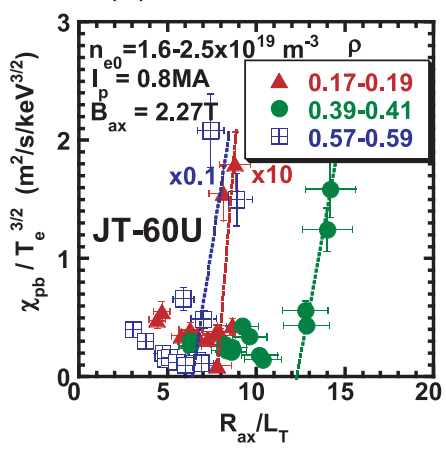

(b)

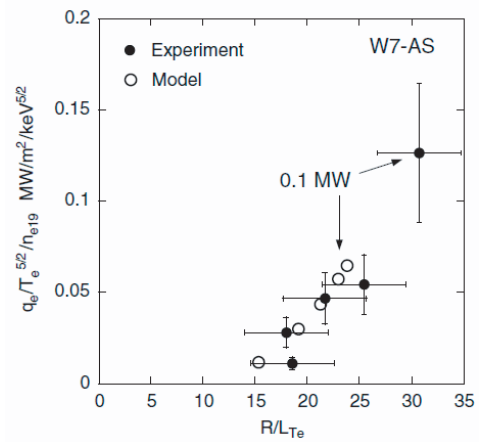

(c)

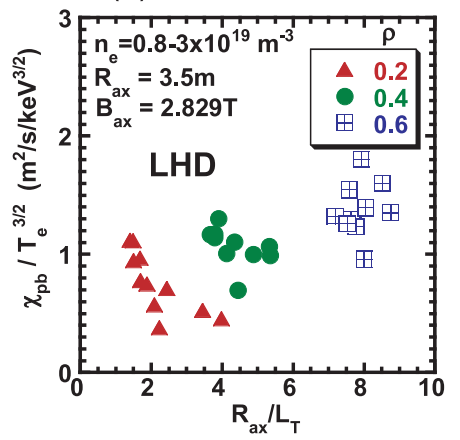

Fig. $6 \nabla T_{\mathrm{e}}$ and $T_{\mathrm{e}}$ dependence of $\chi_{\mathrm{e}}$ at different normalized radii in (a)JT-60U, (b) Wendelstein 7AS, and (c) LHD NBI plasmas. $\chi_{\mathrm{e}}$ is normalized by the gyro-Bohm $T_{\mathrm{e}}$ dependence and $\nabla T_{\mathrm{e}}$ is normalized by $\left(R / T_{\mathrm{e}}\right)$, where $\mathrm{R}$ is the major radius and $L_{T}=\nabla T_{\mathrm{e}} / T_{\mathrm{e}}$ in (a) and (c) $[27,28]$.

clear. $\nabla T_{\mathrm{e}}$ is normalized by $R / T_{\mathrm{e}}$ because the ratio of the major radius to the scale length of the temperature is expected to give the threshold of turbulence in the critical temperature gradient transport model. As seen in Fig. 6, there is no clear critical gradient scale length observed in LHD [27], although the existence of a critical gradient scale length is suggested in Wendelstein 7-AS [28] and JT-60U. The thermal diffusivity $\chi_{\mathrm{e}}$ shows a Gyro-Bohmtype $T_{\mathrm{e}}$ dependence when the temperature gradient is small enough, while it depends mostly on the scale length above a critical value of the temperature gradient. This implies that there is a strong nonlinear mechanism in the transport. One of the candidates for the mechanism causing this strong non-linearity is non-linear flow damping of the zonal flow in a tokamak plasma [29].

In the LHD heliotron, there is no critical value of the temperature gradient, because the transition from the Lmode to an electron ITB takes place at a lower temperature gradient [30]. The transition from L-mode to the electron ITB is due to the change in the temperature dependence of $\alpha$ from a positive value ( 3/2, as predicted by gyroBohm scaling) to negative value $(\sim-3 / 2)$, where $\alpha$ is defined as $Q / n_{\mathrm{e}} \propto T_{\mathrm{e}}^{\alpha} \nabla T_{\mathrm{e}}$ [31]. The temperature dependence tends to decrease (weak temperature dependence) as the electron density is increased. This change in the temperature dependence of the thermal diffusivity is consistent with the change in the power degradation of the energy confinement time. It should be noted that the L-mode scaling of the global energy confinement time expressed by $t_{\mathrm{E}} \propto\left(n_{\mathrm{e}} / P\right)^{3 / 5}$ is equivalent to the temperature dependence of the thermal diffusivity expressed by $\chi \propto T_{\mathrm{e}}^{3 / 2}$. Therefore, the weak temperature dependence $(\alpha=1 / 2)$ appears as a tendency of the saturation of the energy confinement time at higher density as $n_{\mathrm{e}}^{1 / 3}$. The temperature dependence factor $\alpha$ becomes $\sim 0.5$ at lower temperatures below the critical value (in the more collisional regime [32]) or in the plasma with pellet injection [33].

\section{Summary}

In summary, the non-diffusive term driven by the thermo-diffusion due to the temperature gradient is experimentally identified in stellarators and tokamaks. In stellarators, thermo-diffusion always drives outward flux, while that in tokamak it drives inward or outward flux depending on the unstable mode of the turbulence. The inward pinch due to the magnetic field curvature is found in steady-state, fully non-inductive current plasmas in tokamaks. However, this does not explain the inward pinch observed in the heliotron plasma, where the non-diffusive term due to the magnetic field curvature is expected to be outward.

In momentum transport, the significant contribution of the non-diffusive term is experimentally observed and is identified as spontaneous flow. The physics mechanisms driving the spontaneous flow, which is parallel to the magnetic field, are investigated. Both the mean flow perpendicular and parallel to the magnetic field are driven by turbulence as well as by non-ambipolar flux both in tokamak and helical plasmas. The mean flow parallel to the magnetic field (spontaneous toroidal flow) is in the direction of the toroidal flow anti-parallel to the $\left\langle E_{r} \times B_{\theta}\right\rangle$ drift, which is in contrast to the spontaneous flow in a tokamak, where the direction of the toroidal flow is parallel to the direction of the $<E_{r} \times B_{\theta}>$ drift.

Finally, the parameter dependence of the diffusive term of the energy transport is discussed. The thermal diffusivity shows a Gyro-Bohm-type $T_{\mathrm{e}}$ dependence when the temperature gradient is small. When the temperature gradient exceeds the threshold of the scale length, the thermal diffusivity increases rapidly if the plasma stays in the L-mode. The threshold of the scale length is observed in tokamaks and Wendelstein 7-AS plasmas, but not in LHD plasmas, where the transition from the L-mode to the electron ITB mode takes place before the threshold. 


\section{Acknowledgment}

The author would like to thank Drs.K. Tanaka and M. Yoshinuma (NIFS) for fruitful discussions in organizing this paper. The author also would like to thank Drs.C. Hidalgo (CIMAT) and U. Stroth (Stuttgart Univ.), and G.T. Hoang (CEA) for useful discussions on the interpretation of the experimental data in their devices.

[1] A.A. Ware, Phys. Rev. Lett. 25, 15 (1970).

[2] P.W. Terry, Phys. Fluids B 1, 1932 (1989).

[3] U. Stroth et al., Phys. Rev. Lett. 82, 928 (1999).

[4] K. Nagashima et al., Nucl. Fusion 34, 449 (1994).

[5] K. Ida et al., Phys. Rev. Lett. 74, 1990 (1995).

[6] J. Rice et al., Nucl. Fusion 38, 75 (1998).

[7] B. Coppi, Nucl. Fusion 42, 1 (2002).

[8] K. Ida et al., Phys. Rev. Lett. 86, 3040 (2001).

[9] Y. Sakamoto et al., Nucl. Fusion 41, 865 (2001).

[10] S.-I. Itoh, Phys. Fluids B 4, 796 (1992).

[11] O.D. Gurcan, P.H. Diamond et al., Phys. Plasmas 14, 042306 (2007).

[12] K. Tanaka et al., Nucl. Fusion 46, 110 (2006).

[13] G.T. Hoang et al., Phys. Rev. Lett. 93, 135003 (2004).

[14] B. Coppi and C. Spight, Phys. Rev. Lett. 41, 551 (1978).

[15] W. Tang et al., Phys. Fluids 29, 3715 (1986).
[16] M.B. Isichenko, A.V. Gruzinov, and P.H. Diamond, Phys. Rev. Lett. 74, 4436 (1996).

[17] D.R. Baker and M.N. Rosenbluth, Phys. Plasmas 5, 2936 (1998).

[18] Y. Nakamura et al., Nucl. Fusion 43, 219 (2003).

[19] K. Ida et al., Nucl. Fusion 45, 391 (2005).

[20] K. McCormick et al., Phys. Rev. Lett. 89, 015001 (2002).

[21] K. Ida et al., Phys. Rev. Lett. 67, 58 (1991).

[22] K. Ida et al., Plasma Phys. Control. Fusion 44, 361 (2002)

[23] M. Yoshinuma et al., Plasma Fusion Res. 3, S1014 (2008).

[24] C. Hidalgo et al., Plasma Phys. Control. Fusion 48, S169 (2006)

[25] B. Goncalves et al., Phys. Rev. Lett. 96, 145001 (2006).

[26] T.C. Luce et al., Phys. Rev. Lett. 68, 52 (1992).

[27] S. Inagaki, H. Takenaga, K. Ida et al., Nucl. Fusion 46, 133 (2006).

[28] F. Ryter et al., Plasma Phys. Control. Fusion 48, B453 (2006)

[29] P.H. Diamond, S-I. Itoh, K. Itoh and T.S. Hahm, Plasma Phys. Control. Fusion 47, R35 (2005).

[30] K. Ida et al., Phys. Rev. Lett. 91, 085003 (2003).

[31] K. Ida et al., Phys. Plasmas 11, 2551 (2004).

[32] J. Miyazawa et al., Plasma Phys. Control. Fusion 47, 801 (2005).

[33] K. Ida et al., Phys. Rev. Lett. 96, 1250063 (2006). 